\title{
Efecto en las propiedades mecánicas de daños superficiales generados en tubería compuesta flexible para transporte de hidrocarburos
}

The Effect of Superficial Damage on the Mechanical Properties of Flexible Composite Pipe for Transporting Hydrocarbons

\author{
Luz Amparo Quintero Ortiz ${ }^{(1)}$, Javier Ricardo Arciniegas Villamizar ${ }^{(2)}$, María Cathalina Jiménez Romero ${ }^{(3)}$, Albert Yesid Vallen \\ Vargas ${ }^{(4)}$ \\ (1) Magíster en Ingeniería Metalúrgica. Profesora asociada, Escuela de Ingeniería Metalúrgica y Ciencia de Materiales, Facultad de Inge- \\ nierías Fisicoquímicas, Universidad Industrial de Santander, Bucaramanga, Colombia. Directora del Grupo de Desarrollo y Tecnología \\ de Nuevos Materiales (GIMAT) de la Universidad Industrial de Santander. luzquint@uis.edu.co \\ (2) Magíster en Ingeniería de Materiales. Escuela de Ingeniería Metalúrgica y Ciencia de Materiales, Facultad de Ingenierías Fisicoquí- \\ micas, Universidad Industrial de Santander, Bucaramanga, Colombia. Grupo de Desarrollo y Tecnología de Nuevos Materiales de la \\ Universidad Industrial de Santander GIMAT. javier6@gmail.com \\ (3) Ingeniera Metalúrgica. Escuela de Ingeniería Metalúrgica y Ciencia de Materiales, Facultad de Ingenierías Fisicoquímicas, Universidad \\ Industrial de Santander, Bucaramanga, Colombia. Grupo de Desarrollo y Tecnología de Nuevos Materiales de la Universidad Industrial \\ de Santander GIMAT. catabass9@hotmail.com \\ (4) Ingeniero Metalúrgico. Escuela de Ingeniería Metalúrgica y Ciencia de Materiales, Facultad de Ingenierías Fisicoquímicas, Universidad \\ Industrial de Santander, Bucaramanga, Colombia. Grupo de Desarrollo y Tecnología de Nuevos Materiales de la Universidad Industrial \\ de Santander GIMAT. albert_eng724@hotmail.com
}

Recibido 17 de noviembre de 2014. Modificado 11 de junio de 2015. Aprobado 24 de junio de 2015.

DOI: http://dx.doi.org/10.16924/riua.v0i42.756

\section{Palabras clave}

Propiedades mecánicas, rayaduras, sistema de tubería compuesta flexible.

\section{Resumen}

Este trabajo estudió la influencia de rayaduras inducidas superficialmente en la capa externa en las propiedades mecánicas de dos sistemas de tubería compuesta flexible con refuerzo no metálico utilizadas para el transporte de hidrocarburos. Se realizaron ensayos de tracción por anillo muescado, dureza Shore D, y caracterización física por microscopía cofocal sobre muestras con y sin daño superficial, teniendo en cuenta normas y especificaciones técnicas internacionales. Los resultados muestran que la variación de la profundidad y el espaciamiento entre rayaduras afectan las propiedades mecánicas, disminuyendo el esfuerzo último a la tensión y la deformación unitaria, siendo mayor la influencia de la profundidad de las mismas.

\section{Key words}

Mechanical properties, scratches, flexible composite pipe systems.

\begin{abstract}
This research examines the influence of outer sheath scratching on the mechanical properties of two systems of flexible composite pipe with non-metal-reinforcement for transporting hydrocarbons. Tensile testing was carried out on notched rings, using Shore D hardness testing, and by physical characterization using cofocal microscopy on specimens with and without superficial damage. The tests were conducted following international technical standards and specifications. The results showed that variation in the depth and spacing of scratches affect the mechanical properties of the pipe, reducing ultimate tensile strength and leading to unit deformation. The effects are greater the deeper the scratches.
\end{abstract}

\section{INTRODUCCIÓN}

Las tuberías compuestas flexibles utilizadas en la industria del Petróleo y Gas, como alternativa de reemplazo a la tubería metálica, con el fin de minimizar costos causados por la corrosión y el mantenimiento, están comercialmente disponibles para diferentes servicios de fluidos, transporte de mezclas multifásicas crudo-agua-gas, suministro de gas combustible y líneas de distribución, y, para inyección de 
agua en campos de producción o tuberías de disposición (Duarte \& Manrique, 2012). Generalmente, las tuberías están conformadas por tres capas, cuyas propiedades influyen en el comportamiento y aplicabilidad final. Cada capa tiene una función particular. La capa interna de material polimérico contiene el fluido transportado, es resistente a la corrosión y a los hidrocarburos y tiene baja permeabilidad. La capa intermedia o estructural, que envuelve la capa interna de contención, proporciona la resistencia mecánica requerida para soportar las cargas aplicadas durante la instalación y la operación, y cuando el refuerzo es no metálico puede estar constituida por fibras longitudinales o trenzadas, según se requiera capacidad de tensión o resistencia a la presión. La capa externa o cubierta protege el refuerzo estructural o capa intermedia (Dalmolen, 2006).

Pese a las grandes ventajas ofrecidas por estos sistemas de tubería, nuevas investigaciones (Arciniegas, 2011; Duarte \& Manrique, 2012; Arciniegas, Quintero \& Meneses, 2013) han demostrado que estas fallan y que, a menudo, estas fallas son completamente inesperadas o inusuales. Su causa principal es el daño generado por terceros que puede manifestarse por rayaduras y abolladuras, entre otros (Ezrin \& Lavigne, 2006; Farshad, 2006). Estos daños producen cambio de sus propiedades mecánicas y, por tanto, generan pérdidas de hidrocarburo atribuidas a fugas.

Debido a la frecuencia de ocurrencia de las rayaduras en este tipo de tuberías, asociadas con procesos de almacenamiento, transporte e instalación de estas (Arciniegas, 2011; Duarte \& Manrique, 2012), se establecieron las rayaduras como el mecanismo de daño a trabajar en esta investigación. Con el propósito de estudiar el efecto sobre las propiedades mecánicas de la presencia de rayaduras inducidas artificialmente en la capa externa, se utilizaron en este trabajo dos sistemas de tubería compuesta flexible sin refuerzo metálico, las cuales son empleadas en líneas de conducción de crudo e inyección de agua (Dalmolen, 2006; Duarte \& Manrique, 2012) en la Empresa Colombiana de Petróleos (ECOPETROL S.A), quien suministró las muestras. Para ello, se indujeron rayaduras sobre las muestras seleccionadas y se analizaron las propiedades mecánicas en muestras con y sin daño para comparación.

\section{Metodologia}

Para el desarrollo de la investigación se establecieron como variables de afectación el espaciamiento entre rayaduras (2,0 mm y 3,0 mm), la profundidad de la rayadura (10\% y 50\% respecto al espesor de la capa externa) y el sistema de tubería compuesta flexible (tipo I y II). Adicionalmente, se indujeron rayaduras a las muestras de manera artificial y, luego, las muestras fueron probadas con y sin daño, por el ensayo de tensión por anillo muescado, obteniéndose información sobre el efecto de estas variables sobre la deformación unitaria $(\varepsilon)$, el grado de rigidez y el esfuerzo último a la tensión (UTS). Por otra parte, se creó un diseño de experimentos factorial $2^{\mathrm{n}}$ con dos niveles de afectación (alto y bajo) por cada variable y se utilizó un software estadístico para evaluar la influencia de las variables y sus interacciones sobre las propiedades mecánicas. El procedimiento experimental para la investigación se llevó a cabo en tres fases:

\section{Fase 1: Caracterización DE TUbería COMPUESTa FLEXIBLE NO AFECTADA}

Se decidió trabajar con dos sistemas de tubería, los cuales fueron suministrados por ECOPETROL S.A., utilizando criterios técnicos, económicos y aprovechando la facilidad para adquirir muestras de tuberías compuestas flexibles más utilizadas para el transporte de hidrocarburos en los campos petroleros en Colombia. En la Tabla 1 se presentan detalles

\begin{tabular}{|c|c|c|c|c|c|c|}
\hline \multirow[b]{2}{*}{$\begin{array}{l}\text { Sistema de } \\
\text { tubería } \\
\text { compuesta } \\
\text { flexible }\end{array}$} & \multicolumn{2}{|c|}{ Información técnica } & \multicolumn{3}{|c|}{ Constitución tricapa } & \\
\hline & $\begin{array}{l}\text { Diámetro } \\
\text { (in) }\end{array}$ & $\begin{array}{l}\text { Presión máx. } \\
\text { de operación } \\
\text { (psi) }\end{array}$ & $\begin{array}{c}\text { Capa interna } \\
{[1]}\end{array}$ & $\begin{array}{c}\text { Capa intermedia } \\
\text { (malla de } \\
\text { refuerzo) [2] }\end{array}$ & $\begin{array}{c}\text { Capa externa } \\
{[3]}\end{array}$ & Tecnología \\
\hline Sistema I & $23 / 8$ & 275 & $\begin{array}{l}\text { Nylon (PA) } \\
\text { Polipropileno } \\
\text { Fortron } \\
\text { (PPS) }\end{array}$ & Fibra de Aramida & $\begin{array}{c}\text { Nylon (PA) } \\
\text { Polipropileno (PP) }\end{array}$ & \\
\hline Sistema II & 3,0 & 1000 & $\begin{array}{l}\text { Polietileno de Alta } \\
\text { Densidad (PEAD) }\end{array}$ & $\begin{array}{l}\text { Fibra de Poliestireno } \\
\text { (PET) }\end{array}$ & $\begin{array}{c}\text { Polietileno de Alta } \\
\text { Densidad (PEAD) }\end{array}$ & \\
\hline
\end{tabular}

Tabla 1. Características de los sistemas de tubería compuesta flexible seleccionados. Fuente: Elaboración propia. 
de estos dos sistemas; a las muestras se les realizó inspección visual para verificar su coloración y sanidad.

De los sistemas de tubería compuesta flexible seleccionados, se prepararon tres muestras en forma de anillo muescado a partir de tubería sin ninguna afectación, con el fin de evaluar su resistencia a la tensión aparente. Se tuvieron en cuenta los criterios establecidos en la norma técnica ASTM D2290-12 (procedimiento A), en cuanto a las dimensiones de las probetas. Además, se diseñaron y se fabricaron las mordazas y accesorios indicados por la norma para llevar a cabo el ensayo. Así mismo, se realizó la prueba de dureza superficial Shore D en la capa externa. Para llevar a cabo la prueba de dureza se seleccionó al azar una muestra de anillo muescado para cada sistema de tubería. Este proceso se realizó bajo los lineamientos de la norma técnica ASTM D2240-05, así como de la especificación API Spec 17J, y se utilizó un durómetro análogo con indentador tipo D disponible en ECOPETROL S.A. Estas pruebas se adelantaron para obtener información del material, la cual no es suministrada por el fabricante, y es necesaria para hacer comparaciones con la tubería afectada.

\section{FASE 2: OBTENCIÓN Y CARACTERIZACIÓN DE} MUESTRAS CON DAÑO SUPERFICIAL SIMULADO

En esta fase se realizó la actividad de afectación de muestras de tubería compuesta flexible sin defectos por inducción de rayaduras sobre la capa externa. Para la elaboración de las rayas se tuvieron en cuenta algunos parámetros descritos en la norma ASTM D7027-05, tales como la velocidad de rayado, la geometría de la rayadura y la distancia entre rayaduras. La profundidad de las rayaduras se determinó experimentalmente, de tal manera que no se afectara la segunda capa del material compuesto. Así mismo, se realizó la caracterización y la evaluación física de las muestras afectadas mediante microscopía cofocal.

Con el fin de evaluar el efecto de las rayaduras sobre las propiedades mecánicas de las tuberías compuestas flexibles, se utilizaron variables como el espaciamiento entre rayaduras $(2,0$ y 3,0 mm), la profundidad de rayado (10\% y $50 \%$ del espesor de la capa externa) y el sistema de tubería (tipo
I y tipo II). Adicionalmente, debido a las múltiples variables que influyen en el rayado, se establecieron ocho parámetros fijos de operación: el ancho de la rayadura $(0,5 \mathrm{~mm})$, la densidad de rayaduras (cuatro), la orientación de las rayaduras (longitudinal), la ubicación de las rayaduras (centradas en la sección reducida del anillo), la longitud de las rayaduras (acorde con el espaciamiento), la geometría de la rayadura (angular), el material de rayado (acero rápido aleado con cobalto) y la velocidad de avance del rayado (20,0 mm/s). Todas las muestras fueron rayadas en un torno convencional de mecanizado industrial, por medio de un buril 3/8" y bajo condiciones controladas de operación, previa calibración del equipo.

Una vez finalizada la afectación de las muestras, se llevó a cabo la caracterización de las rayaduras mediante un microscopio digital HIROX KH-7700, perteneciente al grupo de investigación en Desarrollo y Tecnología de Nuevos Materiales (GIMAT) de la Universidad Industrial de Santander, con el cual se obtuvieron reconstrucciones tridimensionales e imágenes de las rayaduras inducidas en la capa externa. Se seleccionaron cuatro probetas de anillo muescado para cada sistema de tubería compuesta flexible con diferentes condiciones de rayado en la capa, las cuales fueron observadas al microscopio en aumentos de 140x, 70x y 35x, en campo oscuro y normal respectivamente.

\section{FAse 3: Aplicación Del ensayo De Resistencia A LA TENSIÓN POR ANILLO MUESCADO}

En esta fase se usó una máquina de ensayos universal marca AutoGraph (AG-I Shimadzu), perteneciente al laboratorio de Caracterización de Materiales de la Universidad Santo Tomás de Aquino (USTA - Seccional Bucaramanga). Se hicieron tres pruebas por cada condición de ensayo para tubería compuesta flexible afectada (12 anillos muescados por cada sistema de tubería) (ver Tabla 2), siguiendo la norma ASTM D2290-12 (procedimiento A), el cual determina la tensión aparente de plásticos tubulares y plásticos reforzados. Los parámetros obtenidos fueron: deformación unitaria, esfuerzo último a la tensión y grado de rigidez.

\begin{tabular}{|c|c|c|c|c|}
\hline $\begin{array}{l}\text { Número de anillos } \\
\text { afectados }\end{array}$ & Distribución & $\begin{array}{l}\text { Profundidad de las } \\
\text { rayaduras }\end{array}$ & No. anillos por ensayo & $\begin{array}{l}\text { Espaciamiento entre } \\
\text { rayaduras }\end{array}$ \\
\hline \multirow{4}{*}{12} & \multirow[b]{2}{*}{6} & \multirow{2}{*}{$\begin{array}{c}10 \% \\
\text { (Sistema I: 0,24 mm; } \\
\text { Sistema II: 0,34 mm) }\end{array}$} & 3 & $2,0 \mathrm{~mm}$ \\
\hline & & & 3 & $3,0 \mathrm{~mm}$ \\
\hline & \multirow[b]{2}{*}{6} & \multirow{2}{*}{$\begin{array}{c}50 \% \\
\text { (Sistema I: } 1,22 \mathrm{~mm} ; \\
\text { Sistema II: } 1,68 \mathrm{~mm} \text { ) }\end{array}$} & 3 & $2,0 \mathrm{~mm}$ \\
\hline & & & 3 & $3,0 \mathrm{~mm}$ \\
\hline
\end{tabular}

Tabla 2. Número de muestras afectadas. Fuente: Elaboración propia. 


\section{Resultados Y Discusión}

Después de aplicar la metodología descrita anteriormente, se obtuvieron los siguientes resultados:

\section{FASE 1: CARACTERIZACIÓN DE TUBERÍA COMPUESTA FLEXIBLE NO AFECTADA.}

En las Tablas 3 y 4 se indican los resultados obtenidos en los ensayos de resistencia a la tracción por anillo muescado y dureza Shore D, para los dos sistemas de tubería compuesta flexible en estudio.

Los resultados obtenidos en el ensayo de dureza Shore D permiten establecer que la capa externa correspondiente al sistema de tubería I es más resistente a la penetración, en comparación con la capa externa del sistema II. Esto se debe a la diferencia en el material componente de la capa externa en cada uno de los sistemas, ya que, el Polipropileno (PP) es de mayor dureza que el Polietileno de Alta Densidad (PEAD) (Nicholson, 2006).

\section{FASE 2: OBTENCIÓN DE MUESTRAS CON DAÑO SUPERFICIAL SIMULADO}

En la Tabla 5 se presentan imágenes de los resultados obtenidos de las rayaduras inducidas en cada sistema de tubería compuesta flexible.

Bajo las mismas condiciones, en el sistema II se obtuvo una profundidad de rayadura mayor, lo cual muestra una mayor facilidad de penetración y alta ductilidad para este material. El comportamiento del sistema I se debe probablemente a la composición de la capa externa, la cual está fabricada en polipropileno. Por efecto del rayado, se aprecia una notoria decoloración en las zonas de mayor deformación, con desprendimiento de material y presencia de mayor altura de hombros. Este fenómeno fue significativo para el sistema I, lo cual es característico de la rigidez y de la poca recuperación que presenta el material. Para el sistema II, no hay presencia de hombros, lo cual es representativo de materiales dúctiles y con buena capacidad de deformación elástica, debido a las propiedades típicas del polietileno de alta densidad (Jiang, Browning \& Sue, 2006; Rosa, Sánchez, \& González, 2008).

En cuanto a la forma superficial de la rayadura, el fondo del surco para el sistema I mostró marcas de conchas de mar. Esto sugiere que, a medida que avanza la rayadura, el material se estira en la dirección del rayado y se producen pequeñas grietas perpendiculares a la dirección del mismo. Esto podría dar inicio al crecimiento de grietas a lo largo de la tubería (Jiang, Browning, \& Sue, 2006; Rosa, Sánchez, \& González, 2008). Para el sistema II, se aprecia remoción y deslizamiento de partículas procedentes de la matriz de polietileno de alta densidad.

En cuanto a la forma superficial de la rayadura, el fondo del surco para el sistema I mostró marcas de conchas de mar. Esto sugiere que, a medida que avanza la rayadura, el material se estira en la dirección del rayado y se producen pequeñas grietas perpendiculares a la dirección del mismo. Esto podría dar inicio al crecimiento de grietas a lo largo de la tubería (Jiang, Browning, \& Sue, 2006; Rosa, Sánchez, \& González, 2008). Para el sistema II, se aprecia remoción y deslizamiento de partículas procedentes de la matriz de polietileno de alta densidad.

\begin{tabular}{c|c|c|}
\multirow{2}{*}{$\begin{array}{c}\text { Propiedades } \\
\text { mecánicas }\end{array}$} & \multicolumn{2}{|c|}{ Sistema de tubería compuesta flexible } \\
\cline { 2 - 3 } & Sistema I & Sistema II \\
\hline $\begin{array}{c}\text { Deformación } \\
\text { unitaria }(\varepsilon)\end{array}$ & $0,50 \pm 0,03$ & $1,45 \pm 0,18$ \\
\hline $\begin{array}{c}\text { Grado de rigidez } \\
\text { (Lbf/in) }\end{array}$ & $9027,33 \pm 574,12$ & $7203,72 \pm 510,00$ \\
\hline $\begin{array}{c}\text { Esfuerzo Último a } \\
\text { la Tensión (UTS) } \\
\text { (psi) }\end{array}$ & $3658,29 \pm 349,97$ & $2896,58 \pm 208,20$ \\
\hline
\end{tabular}

Tabla 3. Propiedades mecánicas promedio obtenidas para sistema de tubería no afectada.

Fuente: Elaboración propia.

\begin{tabular}{|c|c|c|c|c|c|c|c|c|c|c|c|}
\hline \multicolumn{12}{|c|}{ Condiciones estándar de ensayo } \\
\hline \multicolumn{5}{|c|}{ Carga: $44,64 \mathrm{~N}$} & Tiempo: $25 \mathrm{~s}$ & \multicolumn{5}{|c|}{ Temperatura: $24^{\circ} \mathrm{C}$} & Humedad: $58 \%$ \\
\hline \multicolumn{12}{|c|}{ Sistema de tubería compuesta flexible } \\
\hline \multicolumn{6}{|c|}{ Sistema I } & \multicolumn{6}{|c|}{ Sistema II } \\
\hline \multicolumn{5}{|c|}{$\begin{array}{l}\text { Puntos de medida de } \\
\text { Dureza (Shore D) }\end{array}$} & \multirow[t]{2}{*}{$\begin{array}{l}\text { Dureza }_{\text {prom }} \\
\text { (Shore D) }\end{array}$} & \multicolumn{5}{|c|}{$\begin{array}{l}\text { Puntos de medida de } \\
\text { Dureza (Shore } D)\end{array}$} & \multirow[t]{2}{*}{$\begin{array}{l}\text { Dureza }_{\text {prom }} \\
\text { (Shore D) }\end{array}$} \\
\hline 1 & 2 & 3 & 4 & 5 & & 1 & 2 & 3 & 4 & 5 & \\
\hline 66,20 & 70,20 & 69,20 & 65,30 & 66,50 & $67,48 \pm 2,10$ & 56,80 & 52,10 & 54,80 & 61,40 & 56,50 & $56,32 \pm 3,40$ \\
\hline
\end{tabular}




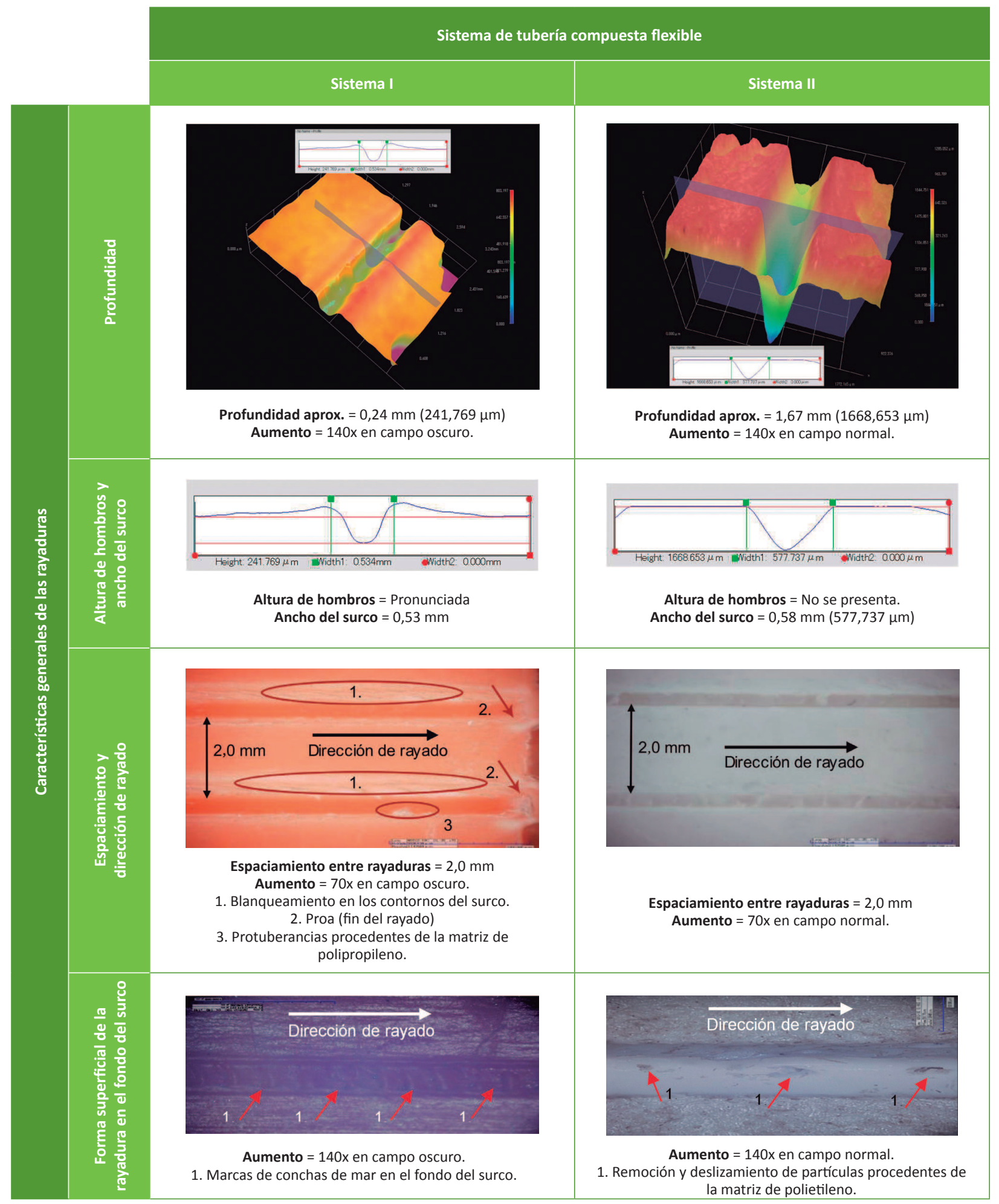

Tabla 5. Características generales de las rayaduras inducidas en los sistemas de tubería.

Fuente: Elaboración propia. 
FAse 3: Aplicación del ensayo de Resistencia a LA TENSIÓN POR ANILLO MUESCADO

Los valores obtenidos en esta fase para la tubería compuesta flexible afectada, de los dos sistemas estudiados, se muestran en la Tabla 6. En esta, se presentan los valores promedio de tres ensayos realizados para cada propiedad mecánica evaluada.
Los datos de las propiedades mecánicas obtenidos experimentalmente se analizaron mediante el software estadístico, con un nivel de confiabilidad del $95 \%$ en el manejo de datos. En las Tablas 7 y 8 se presentan los datos y el análisis de varianza obtenidos por el software para cada propiedad mecánica evaluada.

\begin{tabular}{|c|c|c|}
\hline Sistema de tubería compuesta flexible & Sistema I & Sistema II \\
\hline Área sección transversal del anillo & $0,1322\left(\mathrm{in}^{2}\right)$ & $0,2828\left(\mathrm{in}^{2}\right)$ \\
\hline Longitud inicial (sección reducida) (Lo) & 0,70 (in) & 0,70 (in) \\
\hline Combinación de variables & Todas las variables en el nivel bajo & $\begin{array}{l}\text { Sistema de tubería en nivel alto; } \\
\text { profundidad y espaciamiento nivel bajo }\end{array}$ \\
\hline Profundidad de la rayadura & $10 \%$ espesor de la capa externa $(0,24 \mathrm{~mm})$ & $10 \%$ espesor de la capa externa $(0,34 \mathrm{~mm})$ \\
\hline Espaciamiento entre rayaduras & $2,0 \mathrm{~mm}$ & $2,0 \mathrm{~mm}$ \\
\hline Propiedad mecánica & Valor promedio & Valor promedio \\
\hline Deformación unitaria $(\varepsilon)$ & $0,48 \pm 0,02$ & $1,08 \pm 0,14$ \\
\hline Grado de rigidez (Lbf/in) & $9282,74 \pm 632,93$ & $7763,27 \pm 311,88$ \\
\hline Esfuerzo último a la tensión (UTS) (psi) & $3605,15 \pm 17,42$ & $2781,09 \pm 209,79$ \\
\hline Combinación de variables & $\begin{array}{l}\text { Profundidad en nivel alto; espaciamiento } \\
\text { y sistema de tubería en nivel bajo }\end{array}$ & $\begin{array}{l}\text { Sistema de tubería y profundidad nivel } \\
\text { alto; espaciamiento en nivel bajo }\end{array}$ \\
\hline Profundidad de la rayadura & $50 \%$ espesor de la capa externa $(1,22 \mathrm{~mm})$ & $50 \%$ espesor de la capa externa $(1,68 \mathrm{~mm})$ \\
\hline Espaciamiento entre rayaduras & $2,0 \mathrm{~mm}$ & $2,0 \mathrm{~mm}$ \\
\hline Propiedad mecánica & Valor promedio & Valor promedio \\
\hline Deformación unitaria $(\varepsilon)$ & $0,46 \pm 0,05$ & $1,25 \pm 0,04$ \\
\hline Grado de rigidez (Lbf/in) & $9820,90 \pm 776,32$ & $7886,19 \pm 209,96$ \\
\hline Esfuerzo último a la tensión (UTS) (psi) & $3117,20 \pm 127,20$ & $2495,03 \pm 81,21$ \\
\hline Combinación de variables & $\begin{array}{l}\text { Espaciamiento nivel alto; profundidad y } \\
\text { sistema de tubería en nivel bajo }\end{array}$ & $\begin{array}{l}\text { Sistema de tubería y espaciamiento en } \\
\text { nivel alto; profundidad en nivel bajo }\end{array}$ \\
\hline Profundidad de la rayadura & $10 \%$ espesor de la capa externa $(0,24 \mathrm{~mm})$ & $10 \%$ espesor de la capa externa $(0,34 \mathrm{~mm})$ \\
\hline Espaciamiento entre rayaduras & $3,0 \mathrm{~mm}$ & $3,0 \mathrm{~mm}$ \\
\hline Propiedad mecánica & Valor promedio & Valor promedio \\
\hline Deformación unitaria $(\varepsilon)$ & $0,47 \pm 0,06$ & $1,12 \pm 0,18$ \\
\hline Grado de rigidez (Lbf/in) & $9376,85 \pm 7,21$ & $7280,78 \pm 549,34$ \\
\hline Esfuerzo último a la tensión (UTS) (psi) & $3616,66 \pm 175,45$ & $2600,18 \pm 445,22$ \\
\hline Combinación de variables & $\begin{array}{l}\text { Profundidad y espaciamiento en nivel } \\
\text { alto; sistema de tubería en nivel bajo }\end{array}$ & Todas las variables en el nivel alto \\
\hline Profundidad de la rayadura & $50 \%$ espesor de la capa externa $(1,22 \mathrm{~mm})$ & $50 \%$ espesor de la capa externa $(1,68 \mathrm{~mm})$ \\
\hline Espaciamiento entre rayaduras & $3,0 \mathrm{~mm}$ & $3,0 \mathrm{~mm}$ \\
\hline Propiedad mecánica & Valor promedio & Valor promedio \\
\hline Deformación unitaria $(\varepsilon)$ & $0,43 \pm 0,07$ & $1,07 \pm 0,10$ \\
\hline Grado de rigidez (Lbf/in) & $9819,67 \pm 774,20$ & $6867,66 \pm 505,99$ \\
\hline Esfuerzo último a la tensión (UTS) (psi) & $3041,04 \pm 93,00$ & $2174,20 \pm 316,25$ \\
\hline
\end{tabular}

Tabla 6. Propiedades mecánicas promedio obtenidas para los sistemas de tubería compuesta flexible afectada. Fuente: Elaboración propia. 


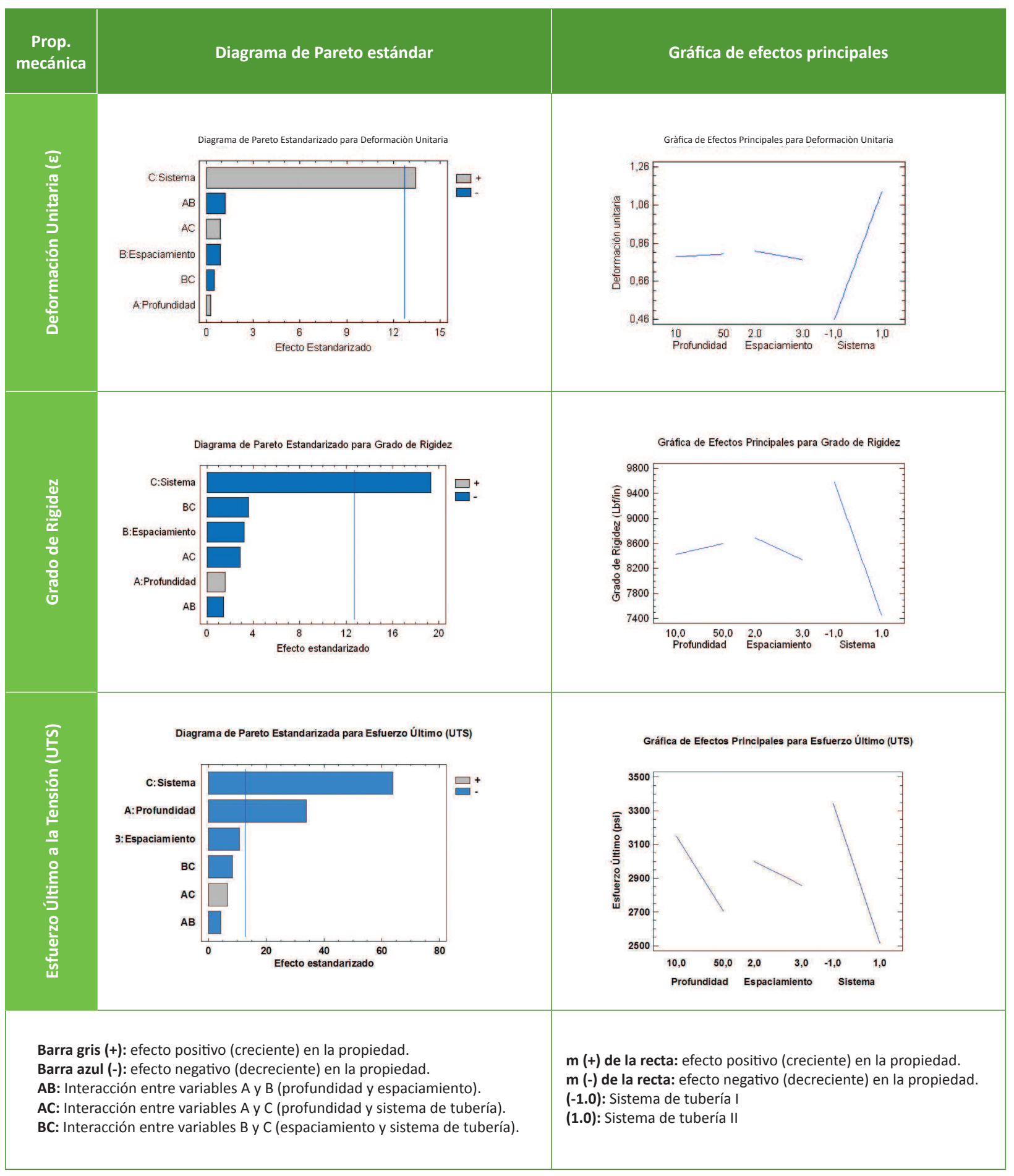

Tabla 7. Gráficas de Pareto y efectos principales para cada propiedad mecánica.

Fuente: Elaboración propia. 


\begin{tabular}{|c|c|c|c|c|c|}
\hline \multicolumn{6}{|c|}{ Análisis de Varianza para Deformación Unitaria } \\
\hline Fuente & Suma de Cuadrados & Grados de Libertad & Cuadrado Medio & Razón-F & Valor-P \\
\hline A: Profundidad & 0,00045 & 1 & 0,00045 & 0,09 & 0,8145 \\
\hline B: Espaciamiento & 0,00405 & 1 & 0,00405 & 0,81 & 0,5335 \\
\hline C: Sistema de Tubería & 0,8978 & 1 & 0,8978 & 179,56 & 0,0474 \\
\hline AB & 0,0072 & 1 & 0,0072 & 1,44 & 0,4423 \\
\hline $\mathrm{AC}$ & 0,00405 & 1 & 0,00405 & 0,81 & 0,5335 \\
\hline BC & 0,00125 & 1 & 0,00125 & 0,25 & 0,7048 \\
\hline Error total & 0,005 & 1 & 0,005 & & \\
\hline Total (corr.) & 0,9198 & 7 & & & \\
\hline R-cuadrada & \multicolumn{5}{|c|}{$99,4564 \%$} \\
\hline $\begin{array}{l}\text { R-cuadrada (ajustada por grados de } \\
\text { libertad) }\end{array}$ & \multicolumn{5}{|c|}{$96,1948 \%$} \\
\hline Error estándar del estimado & \multicolumn{5}{|c|}{0,0707107} \\
\hline \multicolumn{6}{|c|}{ Análisis de Varianza para Grado de Rigidez } \\
\hline Fuente & Suma de Cuadrados & Grados de Libertad & Cuadrado Medio & Razón-F & Valor-P \\
\hline A: Profundidad & 59647,1 & 1 & 59647,1 & 2,46 & 0,3615 \\
\hline B: Espaciamiento & 247857,0 & 1 & 247857,0 & 10,21 & 0,1931 \\
\hline C: Sistema de Tubería & $9,03605 * 10^{6}$ & 1 & $9,03605 * 10^{6}$ & 372,21 & 0,0330 \\
\hline AB & 49830,1 & 1 & 49830,1 & 2,05 & 0,3879 \\
\hline $\mathrm{AC}$ & 201987,0 & 1 & 201987,0 & 8,32 & 0,2125 \\
\hline BC & 317565,0 & 1 & 317565,0 & 13,08 & 0,1717 \\
\hline Error total & 24277,1 & 1 & 24277,1 & & \\
\hline Total (corr.) & $9,93722 * 10^{6}$ & 7 & & & \\
\hline R-cuadrada & \multicolumn{5}{|c|}{$99,7557 \%$} \\
\hline $\begin{array}{l}\text { R-cuadrada (ajustada por grados de } \\
\text { libertad) }\end{array}$ & \multicolumn{5}{|c|}{$98,2899 \%$} \\
\hline Error estándar del estimado & \multicolumn{5}{|c|}{155,811} \\
\hline \multicolumn{6}{|c|}{ Análisis de Varianza para Esfuerzo último a la tensión } \\
\hline Fuente & Suma de Cuadrados & Grados de Libertad & Cuadrado Medio & Razón-F & Valor-P \\
\hline A:Profundidad & 394099,0 & 1 & 394099,0 & 1154,84 & 0,0187 \\
\hline B:Espaciamiento & 40099,7 & 1 & 40099,7 & 117,51 & 0,0586 \\
\hline C:Sistema de Tubería & $1,38574 * 10^{6}$ & 1 & $1,38574 * 10^{6}$ & 4060,68 & 0,0100 \\
\hline AB & 6474,65 & 1 & 6474,65 & 18,97 & 0,1437 \\
\hline $\mathrm{AC}$ & 15446,7 & 1 & 15446,7 & 45,26 & 0,0939 \\
\hline $\mathrm{BC}$ & 23881,0 & 1 & 23881,0 & 69,98 & 0,0757 \\
\hline Error total & 341,258 & 1 & 341,258 & & \\
\hline Total (corr.) & $1,86608 * 10^{6}$ & 7 & & & \\
\hline R-cuadrada & \multicolumn{5}{|c|}{$99,9817 \%$} \\
\hline $\begin{array}{l}\text { R-cuadrada (ajustada por grados de } \\
\text { libertad) }\end{array}$ & \multicolumn{5}{|c|}{$99,872 \%$} \\
\hline Error estándar del estimado & \multicolumn{5}{|c|}{18,4732} \\
\hline
\end{tabular}

Tabla 8. Análisis de Varianza para cada propiedad mecánica. Fuente: Elaboración propia. 
El diagrama de Pareto mostró una influencia significativa del tipo de tubería sobre la deformación unitaria y muy poca influencia de la profundidad y del espaciamiento de rayado, así como de sus interacciones sobre esta propiedad. Lo anterior se corroboró con la respectiva gráfica de efectos principales. Se mostró para esta variable una recta de pendiente positiva, evidenciándose mayor deformación para el sistema de tubería II, lo cual está acorde con las propiedades mecánicas indicadas para este sistema.

En cuanto al efecto de las variables sobre el grado de rigidez, tanto el diagrama de Pareto como la gráfica de efectos principales, mostraron una influencia negativa (decreciente) de la variable sistema de tubería, debido probablemente a la diferencia en propiedades mecánicas de los materiales componentes de estos sistemas. El espaciamiento entre rayaduras y su profundidad no presentaron influencia sobre esta propiedad.

La resistencia última a la tensión se mostró influenciada por el sistema de tubería y la profundidad de rayado. Se observó menor efecto de la variable espaciamiento entre rayaduras.

En el análisis realizado se notó la gran influencia del tipo de tubería sobre los resultados obtenidos, lo cual está de acuerdo con la diferencia en las propiedades mecánicas de los sistemas analizados. Por otra parte, fue más significativa la profundidad de rayado que el espaciamiento entre rayaduras sobre las propiedades mecánicas de la tubería afectada por este daño.

\section{Conclusiones}

A partir de las propiedades mecánicas obtenidas experimentalmente para los sistemas de tubería con y sin afectación, se demostró que el daño inducido por rayaduras en la capa externa, en ambos sistemas, a profundidades de rayado del $10 \%$ y $50 \%$ y espaciamiento entre rayaduras de $2,0 \mathrm{~mm}$ y 3,0 mm, afectó sus propiedades mecánicas, presentándose disminución de la deformación y del esfuerzo último a la tensión. Se apreció un incremento del grado de rigidez del sistema, el cual, se puede asociar a un aumento en la rigidez del material constituyente de la capa externa.

El espaciamiento entre rayaduras no fue significativo en la variación de las propiedades mecánicas para los sistemas de tubería compuesta flexible estudiados en esta investigación, ya que, fue más influyente la profundidad de rayado sobre estas.

La caracterización microscópica permitió corroborar las diferentes características de los materiales estudiados, confirmando con los ensayos de tensión y dureza Shore D, la resistencia a la penetración del material de la capa externa, presentando un comportamiento más dúctil en el caso del sistema de tubería II.

\section{Agradecimientos}

Los autores agradecen al Instituto Colombiano del Petróleo (ECOPETROL S.A - ICP), al grupo de investigación GIMAT de la UIS, al Dr. Bert Dalmolen (Pipelife Nederland B.V), al Ing. Mario Candela Cely (Imantt Solutions Ltda), y a MSc. Pedro Pablo Díaz (USTA - Sede Bucaramanga) por su valiosa asesoría y aporte técnico-científico en el desarrollo de la presente investigación.

\section{REFERENCIAS}

American Petroleum Institute. (2008). Specification for Unbonded Flexible Pipe. ANSI/API Specification 17J, API Spec 17J. doi: 202.682.8000.

American Society for Testing and Materials. (2010). Standard Test Method for Rubber Property-Durometer Hardness. ASTM International, ASTM D2240-05. doi: 10.1520/D2240-05R10.

American Society for Testing and Materials. (2012). Standard Test Method for Apparent Hoop Tensile Strength of Plastic or Reinforced Plastic Pipe. ASTM International, ASTM D2290-12. doi: 10.1520/D2290-12.

American Society for Testing and Materials. (2005). Standard Test Method for Evaluation of Scratch Resistance of Polymeric Coatings and Plastics Using an Instrumented Scratch Machine. ASTM International, ASTM D7027-05. doi: 10.1520/D7027-05E01.

Arciniegas, J. (2011). Instalación de Tuberías No Metálicas en Líneas de Superficie en los pozos del campo la CiraInfantas. Ponencia presentada en el XI Congreso Nacional de Corrosión, Bucaramanga, Colombia.

Arciniegas, J., Quintero, L., \& Meneses, J. (2013). Análisis de Técnicas de Inspección No Destructiva en Tubería Flexible con Refuerzo No Metálico para el Transporte de Hidrocarburos. Conferencia llevada a cabo en el VII Congreso Internacional de Materiales, Medellín, Colombia.

Dalmolen, B. (2006). Reinforced Thermoplastic Pipeline (RTP) Systems. Conferencia llevada a cabo en el XXIII Congreso Internacional de Gas, Amsterdam, Holanda.

Duarte, G. \& Manrique, M. (2012). Guía Recomendada para Selección y Uso de Tuberías de Materiales Compuestos para aplicación en Líneas de Flujo de la VPR (Proyecto Infraestructura Segura y Confiable VPR 2012). Piedecuesta, Colombia: ECOPETROL S.A. - Instituto Colombiano del Petróleo ICP - Unidad de Disciplinas Especializadas UDE - Vicepresidencia de Producción VPR - Corporación CIMA.

Ezrin, M. \& Lavigne, G. (2006). Unexpected and Unusual Failures of Polymeric Materials. Proceedings of Second 
International Conference on Engineering Failure Analysis 14(6), 1153-1165. doi:10.1016/j.engfailanal.2006.11.048

Farshad, M. (2006). Plastic Pipe Systems: Failure investigation and diagnosis. Reino Unido: Editorial Elsevier Science Technology.

Jiang, H., Browning. R. \& Sue., H. (2006). Understanding of Scratch - Induced Damage Mechanisms in Polymers. Polymer, 50(16), 4056-4065 doi:10.1016/j.polymer.2009.06.061
Nicholson, J. (2006). The Chemistry of Polymers ( $3^{\mathrm{a}}$ ed.). Cambridge, UK: RSC Publishing. doi: $10.1039 / 9781847552655$

Rosa, A., Sánchez, M. \& González, F. (2008). Ensayo de rayado de los componentes plásticos bicapa rígido - flexibles usados en el diseño de electrodomésticos y herramientas tipo Soft Touch. Revista de arquitectura, diseño y urbanismo, (5), 284-294. 